\title{
Incidence and multidimensional predictors of occasional and recurrent falls among Malaysian community-dwelling older persons
}

Theng Choon Ooi ${ }^{1}$, Devinder Kaur Ajit Singh ${ }^{1 *}$, Suzana Shahar ${ }^{1}$, Nor Fadilah Rajab', Divya Vanoh², Razinah Sharif ${ }^{1}$ and Maw Pin Tan ${ }^{3,4}$

\begin{abstract}
Background: Falls incidence rate and comprehensive data on factors that predict occasional and repeated falls from large population-based studies are scarce. In this study, we aimed to determine the incidence of falls and identify predictors of occasional and recurrent falls. This was done in the social, medical, physical, nutritional, biochemical, cognitive dimensions among community-dwelling older Malaysians.
\end{abstract}

Methods: Data from 1,763 Malaysian community-dwelling older persons aged $\geq 60$ years were obtained from the LRGS-TUA longitudinal study. Participants were categorized into three groups according to the presence of a single fall (occasional fallers), ztwo falls (recurrent fallers), or absence of falls (non-fallers) at an 18-month follow-up.

Results: Three hundred and nine (17.53\%) participants reported fall occurrences at an 18-month follow-up, of whom 85 (27.51\%) had two or more falls. The incidence rate for occasional and recurrent falls was 8.47 and 3.21 per 100 person-years, respectively. Following multifactorial adjustments, being female (OR: 1.57; $95 \%$ Cl: 1.04-2.36), being single (OR: 5.31; $95 \%$ Cl: 3.36-37.48), having history of fall (OR: 1.86; $95 \%$ Cl: 1.19-2.92) higher depression scale score (OR: 1.10; $95 \%$ Cl: 1.02-1.20), lower hemoglobin levels (OR: 0.90; $95 \%$ Cl: 0.81-1.00) and lower chair stand test score (OR: 0.93; $95 \%$ Cl: 0.87-1.00) remained independent predictors of occasional falls. While, having history of falls (OR: $2.74 ; 95 \% \mathrm{Cl}$ : 1.45-5.19), being a stroke survivor (OR: 8.57; $95 \%$ Cl: 2.12-34.65), higher percentage of body fat (OR: $1.04 ; 95 \%$ Cl: $1.01-$ $1.08)$ and lower chair stand test score (OR: $0.87 ; 95 \% \mathrm{Cl}: 0.77-0.97)$ appeared as recurrent falls predictors.

Conclusions: Having history of falls and lower muscle strength were predictors for both occasional and recurrent falls among Malaysian community-dwelling older persons. Modifying these predictors may be beneficial in falls prevention and management strategies among older persons.

Keywords: Depression, Falls, Hemoglobin, Incidence, Muscle strength, Older persons, Predictors

\footnotetext{
* Correspondence: devinder@ukm.edu.my

${ }^{1}$ Centre for Healthy Ageing and Wellness, Faculty of Health Sciences,

Universiti Kebangsaan Malaysia, Jalan Raja Muda Abdul Aziz, 50300 Kuala Lumpur, Malaysia

Full list of author information is available at the end of the article
}

(C) The Author(s). 2021 Open Access This article is licensed under a Creative Commons Attribution 4.0 International License, which permits use, sharing, adaptation, distribution and reproduction in any medium or format, as long as you give appropriate credit to the original author(s) and the source, provide a link to the Creative Commons licence, and indicate if changes were made. The images or other third party material in this article are included in the article's Creative Commons licence, unless indicated otherwise in a credit line to the material. If material is not included in the article's Creative Commons licence and your intended use is not permitted by statutory regulation or exceeds the permitted use, you will need to obtain permission directly from the copyright holder. To view a copy of this licence, visit http://creativecommons.org/licenses/by/4.0/ The Creative Commons Public Domain Dedication waiver (http://creativecommons.org/publicdomain/zero/1.0/) applies to the data made available in this article, unless otherwise stated in a credit line to the data. 


\section{Background}

The global prevalence of falls was 5,186 $(4,622-5,849)$ per 100,000 people in year 2017 [1]. Falls can lead to debilitating consequences, including injury-related hospitalization, disability and death [2]. Moreover, fall-related injuries in older persons result in increased healthcare costs. The estimated medical costs of fatal and non-fatal falls among older persons in the US was reported to be around 50 billion dollars [3]. Population-wide falls prevention programs that effectively improve the overall health and reduce social care burden associated with fall-related complications are, therefore, urgently required [4].

Several risk factors have been identified to be associated with falls in older persons. Females and those who are older in age are at higher risk of falls [5]. In the psychosocial context, older persons who live alone, have fewer years of education, or who experience loneliness and depression are more likely to be at risk of falls [6]. Medical illnesses such as arthritis, diabetes, chronic kidney diseases and stroke are among the other risk factors for falls in older persons [6, 7]. Physical frailty, cognitive decline and other geriatric syndromes also contribute to the occurrence of falls in older persons [5].

Declined physical function below a certain threshold, manifesting as muscle weakness and loss of balance are likely to lead to falls in older persons as well [8]. In addition, older persons with cognitive impairment are twice more likely to fall in comparison to those without cognitive impairment [9]. Nutritional deficiencies, such as vitamin $\mathrm{D}$, calcium and inadequate protein intake are linked to osteoporosis, fractures and sarcopenia and, as a result, increase both the risk of falls and its consequences [10]. Environmental factors, for example, poor lighting, clutter in and around the house and inadequate footwear are often contributors to the risk of falls among older persons [5].

It is noteworthy that most risk factors of falls studied among community-dwelling older persons were performed via cross-sectional studies and were inconclusive. In addition, physical, nutritional, biochemical and biomarker status were not examined together within a single study. As falls are multifactorial in nature, identifying the multidimensional predictors of falls risk may be beneficial for early falls prevention strategies in community-dwelling older persons. The objective of this study was to determine the incidence of falls and identify predictors of occasional and recurrent falls. This was done in the social, medical, physical, nutritional, biochemical and cognitive dimensions among community-dwelling older Malaysians.

\section{Methods}

\section{Participants}

This prospective cohort study involved 1,763 participants from the first wave of the Long-term Research Grant
Scheme - Towards Useful Ageing (LRGS-TUA) study who were successfully followed up at 18-months (wave 2). Participants were recruited from four states representing Malaysia's northern, central, southern and east coast zones through a multistage random sampling approach, as reported by Shahar et al. (2016) [11]. Older persons aged 60 years and above, able to walk with or without aids and those who were able to converse in Malay, English, Chinese, or Tamil language participated in the study. Older persons with severe visual, hearing, musculoskeletal or neurological impairments were excluded. This study was approved by the Medical Research and Ethics Committee of Universiti Kebangsaan Malaysia (UKM 1.5.3.5/244/NN-060-2013).

\section{Data collection}

In wave 1 , demographic data and several risk factors were assessed as described in the previous study [11]. These assessments are described briefly below:

\section{Demographic data}

A standardized questionnaire was used to obtain the socio-demographic and health data from the participants, including age, sex, ethnicity, marital status, living status, years of education, smoking habits, alcohol drinking status and self-reported medical history.

\section{Body composition and blood pressure}

The height, weight and the circumference of the waist, hip and calf were measured using a SECA 206 portable body meter (Seca, Hamburg, Germany), Tanita digital lithium weighing scale (Tanita, Tokyo, Japan) and Lufkin tape respectively, as reported earlier [11]. The body mass index (BMI) of participants were then calculated by using the formula "body weight $(\mathrm{kg}) /$ height $(\mathrm{m})^{2}$. The fat-free mass, fat mass, skeletal muscle mass and percentage of body fat were measured by using the InBody S10 body composition analyzer (Biospace Co. Ltd, Korea). Meanwhile, the systolic and diastolic blood pressure was taken twice using an automatic digital blood pressure monitor (OMRON, Kyoto, Japan) to obtain the average reading.

\section{Dietary Status}

Participants were interviewed using a validated Dietary History Questionnaire for older persons to record their usual dietary intake in a week, as previously described [11]. The nutrient intake was analyzed by using Nutritionist Pro software.

\section{Biochemical analysis}

Fasting venous blood was collected from the participants by a trained phlebotomist and subjected to biochemical analysis. The parameters included in the analysis were 
fasting blood glucose, serum albumin, hemoglobin levels and serum lipid profile [total cholesterol, high-density lipoprotein (HDL), low-density lipoprotein (LDL) and triglyceride levels].

\section{Cognitive status}

The following tests were performed:

\section{i. Mini-Mental State Examination}

General cognitive function was assessed using the Malay version of the Mini-Mental State Examination (MMSE), which is a valid and reliable screening tool for dementia in the Malaysian population [12].

\section{ii. Digit Span}

Digit Span [subtest for the Wechsler Adult Intelligence Scale (WAIS)] is a sensitive tool used to measure working memory [13]. This test is used to assess attention.

\section{iii. Montreal Cognitive Assessment}

The Malay version of the Montreal Cognitive Assessment (MoCA) [14] was used to test for orientation, short memory recall, attention and concentration, working memory and mental arithmetic, language comprehension, visuospatial domain, executive cognitive functioning, naming of objects and abstract thought components.

\section{iv. Digit symbol}

Digit symbol (a subset of WAIS) was used to evaluate visual-motor speed and coordination, visual search and cognitive flexibility [15]. This test requires participants to fill in small blank squares with symbols that matched the number labeled in each square. It is one of the most age-sensitive and useful cognitive tests to distinguish between mild Alzheimer-type dementia and healthy ageing.

\section{Psychosocial}

The validated Malay version of the Geriatric Depression Scale-15 [16] was used to evaluate depression status.

\section{Physical Performance}

\section{i. Timed Up and Go test}

In the Timed Up and Go (TUG) test, participants were required to sit on an armless chair with a seating height of $46 \mathrm{~cm}$. Participants were then instructed to stand up, walk 3 meters at a normal pace, turn around, walk back and sit on the chair. The TUG test was performed twice consecutively and the average time (seconds) to complete the test was recorded. The participants were allowed to walk with their regular walking aids.

\section{ii. 2-minute step test}

The 2-minute step test measures aerobic endurance. Participants were instructed to march on the spot to about $90^{\circ}$ hip flexion and $90^{\circ}$ knee extension. The test was continued for 2 minutes and the number of steps taken were counted.

\section{iii. 30-second chair stand test}

Participants were required to sit on a chair (without armrest) with hands on opposite shoulders crossed at the wrists. They were then instructed to rise to full standing and sitting down again and to repeat this task for 30 seconds. The number of times the sit-to-stand were recorded. This was done twice, with a rest in between. This test reflects lower limb muscle strength.

\section{iv. Dominant handgrip muscle strength test}

The dominant handgrip muscle strength test was performed using a handgrip dynamometer (Jamar Plus+ Hand Dynamometer, SI Instruments Pty Ltd, SA, Australia). The maximum effort was measured twice with shoulder in sustained adduction and neutral rotation, elbow flexed at 90 degrees, forearm neutral and wrist between 0-15 degrees of ulnar deviation. The highest reading was taken as the result.

\section{Falls history at Baseline and follow-up}

During the first wave of the study, participants were asked, "Have you had a fall in the past 12-months?". At the 18-month follow-up interview, participants were asked, "Have you had a fall in the past 18-months?". If they responded positively to the above question, a further question, "How many times have you fallen?" were asked. The participants were informed that a fall is an event whereby a person is inadvertently coming to rest on the ground, floor or other lower levels, excluding intentional change in position to rest on furniture or floor [17]. Participants who reported to have no fall history in the past during follow-up were categorized as non-fallers. Those with one fall were categorized as occasional fallers, while those who reported two or more falls were categorized as recurrent fallers.

\section{Statistical analysis}

The cumulative incidence of falls was calculated by dividing the number of new cases of a fall or recurrent falls at 18-months follow-up with the total number of participants that were successfully followed up at wave 2 
of the study. The incidence rate of falls was calculated by using the person-years method, whereby the number of new cases of a fall or recurrent falls between the two data collection points was computed to the number of cases per 100 person-years. The age-specific incidence rate was then calculated by further dividing the participants into four different age-groups (60-64, 65-69, 7074 and 75 years and above).

Socio-demographic data, body composition, nutrition, biochemical, cognitive and physical performance scores were compared between non-fallers and occasional fallers, as well as non-fallers and recurrent fallers by using the independent $t$-test for continuous variables or Chi-square test for categorical variables. Variables that were found to be significant $(p<0.05)$ in the univariate tests were further analyzed using binary logistic regression analyses. These were then entered into the final multivariate logistic regression analysis using the forward-stepwise logistic model to determine falls predictors. Confounding factors (age, sex, multimorbidity, falls history at baseline, years of education and cognitive performance) were included for adjustment in the multivariate logistic regression model. The statistical analyses were performed using Statistical Package for the Social Sciences (SPSS) version 25 (IBM Corp., Armonk, NY).

\section{Results}

Three hundred and nine (17.53\%) participants reported fall occurrence at an 18-month follow-up, of whom 85 $(27.51 \%)$ had two or more falls. The incidence rate for occasional and recurrent falls was 8.47 and 3.21 per 100 person-years, respectively. The age-specific incidence rates of both occasional and recurrent falls are as shown in Table 1. Overall, the incidence rate of falls was independent of increasing age. The $\geq 75$ age group had the highest incidence rate for occasional falls $(9.09$ per 100 person-years). While the 65-69 years age group had the highest incidence rate for recurrent falls (3.91 per 100 person-years).

Table 2 depicts the baseline characteristics of non, occasional and recurrent fallers. Occasional fallers were more likely $(p<0.05)$ to be women $(60.27 \%)$, single $(3.13 \%)$, have falls history $(30.36 \%)$, have higher depression scale scores $(2.95 \pm 2.42)$; lower fat-free mass
$(35.64 \pm 7.07 \mathrm{~kg})$ and skeletal muscle mass $(18.82 \pm 4.21$ $\mathrm{kg})$; lower intake of energy $(1,583.99 \pm 436.79 \mathrm{kcal} /$ day $)$, carbohydrate $(209.95 \pm 67.82 \mathrm{~g} /$ day $)$ and zinc (3.38 \pm $1.60 \mathrm{mg} /$ day); lower hemoglobin levels $(13.64 \pm 1.85 \mathrm{~g} / \mathrm{L})$, scored lower in MMSE $(22.62 \pm 4.82)$, MoCA (18.35 \pm $5.82)$ and digit symbol $(4.73 \pm 2.50)$; scored lower in chair stand (9.60 \pm 3.28 times) and dominant handgrip muscle strength $(20.82 \pm 7.27 \mathrm{~kg})$ tests. Recurrent fallers were more likely $(p<0.05)$ to be women $(68.24 \%)$, living alone $(17.65 \%)$, have falls history $(42.35 \%)$, with a medical history of stroke (4.71\%) and joint pain (36.47\%); higher depression scale score $(3.35 \pm 2.63)$; lower fat-free mass $(35.17 \pm 7.90 \mathrm{~kg})$ and skeletal muscle mass $(18.51 \pm 4.70$ $\mathrm{kg})$; higher fat mass $(26.32 \pm 10.16 \mathrm{~kg})$ and percentage of body fat $(41.89 \pm 10.26 \%)$; lower hemoglobin levels $(13.42 \pm 2.13 \mathrm{~g} / \mathrm{L})$; scored lower in chair stand $(8.83 \pm 3.07$ times), TUG (12.84 \pm 5.28 seconds) and dominant handgrip muscle strength $(19.57 \pm 6.69 \mathrm{~kg})$ tests.

Variables that were significant in the univariate test were entered into binary logistic regression. The univariate predictors of occasional and recurrent falls are as shown in Table 3. These variables were then further analyzed using multivariate logistic regression analysis (Table 4). Being female (OR: 1.57; $95 \%$ CI: 1.04-2.36), being single (OR: 5.31; $95 \%$ CI: 3.36-37.48), having history of falls (OR: 1.86; $95 \%$ CI: 1.19-2.92) higher depression scale scores (OR: 1.10; $95 \%$ CI: 1.02-1.20), lower hemoglobin levels (OR: 0.90; $95 \%$ CI: 0.81-1.00) and lower chair stand test scores (OR: $0.93 ; 95 \% \mathrm{CI}$ : $0.867-1.00)$ appeared as occasional falls predictors in this model $\left[X^{2} \quad(\mathrm{df}=8, \quad \mathrm{~N}=1763)=13.38, p=0.100\right.$ with $86.60 \%$ accuracy]. While, having history of falls (OR: 2.74; $95 \%$ CI: 1.45-5.19), stroke (OR: 8.57; $95 \%$ CI: 2.12-34.65), higher percentage of body fat (OR: 1.04; $95 \%$ CI: 1.01-1.08) and lower scores in chair stand test (OR: 0.87; $95 \%$ CI: 0.77-0.97) were identified as predictors of recurrent falls $\left[\mathrm{X}^{2} \quad(\mathrm{df}=8, \mathrm{~N}=1763)=10.82\right.$, $p=0.212$ with $94.70 \%$ accuracy].

\section{Discussion}

We identified the incidence rate and multidimensional falls risk predictors at 18-months follow-up among 1,763 community-dwelling older Malaysians. The incidence rate of occasional and recurrent falls observed in our

Table 1 The age-specific incidence rates of occasional falls and recurrent falls over 18-months

\begin{tabular}{|c|c|c|c|c|c|c|c|}
\hline \multirow{2}{*}{$\begin{array}{l}\text { Age } \\
\text { group }\end{array}$} & \multirow{2}{*}{$\begin{array}{l}\text { No. of } \\
\text { Participants }\end{array}$} & \multicolumn{3}{|c|}{ Occasional falls } & \multicolumn{3}{|c|}{ Recurrent falls } \\
\hline & & No. of cases & Cases/year & Cases/100 person-years & No. of cases & Cases/year & Cases/100 person-years \\
\hline $60-64$ & 523 & 64 & 42.67 & 8.16 & 24 & 16.00 & 3.06 \\
\hline $65-69$ & 528 & 68 & 45.33 & 8.59 & 31 & 20.67 & 3.91 \\
\hline 70-74 & 404 & 50 & 33.33 & 8.25 & 14 & 9.33 & 2.31 \\
\hline$\geq 75$ & 308 & 42 & 28.00 & 9.09 & 16 & 10.67 & 3.46 \\
\hline Total & 1,763 & 224 & 149.33 & 8.47 & 85 & 56.67 & 3.21 \\
\hline
\end{tabular}


Table 2 The baseline attributes of the participants with no fall, occasional and recurrent falls

\begin{tabular}{|c|c|c|c|c|c|}
\hline Parameters & $\begin{array}{l}\text { Non-fallers } \\
(n=1454)[\text { Prevalence } \\
(\%) \text { or mean } \pm \text { SD] }\end{array}$ & $\begin{array}{l}\text { Occasional fallers } \\
(n=224)[\text { Prevalence } \\
(\%) \text { or mean } \pm \text { SD] }\end{array}$ & $P$-value & $\begin{array}{l}\text { Recurrent fallers } \\
(n=85)[\text { Prevalence } \\
(\%) \text { or mean } \pm \text { SD] }\end{array}$ & $P$-value \\
\hline Age (years) & $68.62 \pm 5.94$ & $68.80 \pm 6.13$ & 0.668 & $68.65 \pm 6.07$ & 0.967 \\
\hline \multicolumn{6}{|l|}{ Sex } \\
\hline Male & $751(51.65)$ & 89 (39.73) & $0.001^{*}$ & 27 (31.76) & $<0.001^{*}$ \\
\hline Female & $703(48.35)$ & $135(60.27)$ & & $58(68.24)$ & \\
\hline \multicolumn{6}{|l|}{ Ethnicity } \\
\hline Malay & $879(60.45)$ & $146(65.18)$ & 0.119 & $58(68.24)$ & 0.218 \\
\hline Chinese & $509(35.01)$ & $64(28.57)$ & & $22(25.88)$ & \\
\hline Indian & $66(4.54)$ & $14(6.25)$ & & $5(5.88)$ & \\
\hline Single & $18(1.24)$ & $7(3.13)$ & $0.030^{*}$ & $1(1.18)$ & 0.960 \\
\hline Living alone & $138(9.49)$ & $22(9.82)$ & 0.875 & $15(17.65)$ & $0.015^{*}$ \\
\hline Smoking & $251(17.26)$ & $34(15.18)$ & 0.439 & $10(11.76)$ & 0.189 \\
\hline Alcohol consumption & $66(4.54)$ & $7(3.13)$ & 0.334 & $2(2.35)$ & 0.340 \\
\hline Education (years) & $5.38 \pm 4.03$ & $4.88 \pm 3.79$ & 0.083 & $4.54 \pm 4.04$ & 0.063 \\
\hline Falls history & $222(15.27)$ & $68(30.36)$ & $<0.001^{*}$ & $36(42.35)$ & $<0.001^{*}$ \\
\hline \multicolumn{6}{|l|}{ Chronic diseases } \\
\hline Hypertension & $714(49.11)$ & $116(51.79)$ & 0.455 & $46(54.12)$ & 0.369 \\
\hline Diabetes mellitus & $363(24.97)$ & $57(25.45)$ & 0.877 & $24(28.24)$ & 0.499 \\
\hline Stroke & $22(1.51)$ & $3(1.34)$ & 0.842 & $4(4.71)$ & $0.026^{*}$ \\
\hline Joint Pain & 337 (23.18) & $64(28.57)$ & 0.078 & 31 (36.47) & $0.005^{*}$ \\
\hline Cardiovascular diseases & $147(10.11)$ & $21(9.38)$ & 0.733 & $7(8.24)$ & 0.576 \\
\hline Cataract \& glaucoma & $127(8.73)$ & $27(12.05)$ & 0.109 & $7(8.24)$ & 0.874 \\
\hline Asthma & $111(7.63)$ & $18(8.04)$ & 0.834 & $10(11.76)$ & 0.169 \\
\hline Gout & $68(4.68)$ & $7(3.13)$ & 0.295 & $5(5.88)$ & 0.611 \\
\hline Gastric ulcer & $178(12.24)$ & $34(15.18)$ & 0.218 & $15(17.65)$ & 0.144 \\
\hline Urinary Incontinence & $143(9.83)$ & $26(11.61)$ & 0.412 & $11(12.94)$ & 0.354 \\
\hline Hearing \& vision problems & $173(11.90)$ & $23(10.27)$ & 0.479 & $15(17.65)$ & 0.116 \\
\hline \multicolumn{6}{|l|}{ Psychosocial } \\
\hline Depression & $2.54 \pm 2.19$ & $2.95 \pm 2.42$ & $0.012^{*}$ & $3.35 \pm 2.63$ & $0.007^{*}$ \\
\hline \multicolumn{6}{|l|}{ Physical } \\
\hline BMI $\left(\mathrm{kg} / \mathrm{m}^{2}\right)$ & $24.95 \pm 4.41$ & $25.11 \pm 4.83$ & 0.606 & $25.68 \pm 5.07$ & 0.140 \\
\hline Circumference: waist (cm) & $88.21 \pm 11.20$ & $88.08 \pm 11.97$ & 0.870 & $89.88 \pm 12.62$ & 0.239 \\
\hline Circumference: hip (cm) & $96.54 \pm 9.18$ & $96.95 \pm 10.37$ & 0.575 & $98.46 \pm 11.61$ & 0.139 \\
\hline Circumference: calf (cm) & $33.55 \pm 3.75$ & $33.12 \pm 3.85$ & 0.114 & $33.41 \pm 4.08$ & 0.744 \\
\hline Fat mass (kg) & $24.19 \pm 9.09$ & $24.47 \pm 9.67$ & 0.680 & $26.32 \pm 10.16$ & $0.038^{*}$ \\
\hline Fat free mass (kg) & $37.16 \pm 7.89$ & $35.64 \pm 7.07$ & $0.004^{*}$ & $35.17 \pm 7.90$ & $0.025^{*}$ \\
\hline Skeletal muscle mass (kg) & $19.75 \pm 4.71$ & $18.82 \pm 4.21$ & $0.003^{*}$ & $18.51 \pm 4.70$ & $0.019^{*}$ \\
\hline Percentage of body fat (\%) & $38.68 \pm 10.38$ & $39.69 \pm 10.20$ & 0.178 & $41.89 \pm 10.26$ & $0.006^{*}$ \\
\hline Systolic (mmHg) & $140.70 \pm 22.40$ & $141.13 \pm 20.34$ & 0.795 & $138.81 \pm 23.46$ & 0.471 \\
\hline Diastolic (mmHg) & $77.26 \pm 13.44$ & $76.87 \pm 12.96$ & 0.689 & $77.31 \pm 12.88$ & 0.975 \\
\hline \multicolumn{6}{|l|}{ Nutrition } \\
\hline Energy (kcal/day) & $1,658.03 \pm 485.42$ & $1,583.99 \pm 436.79$ & $0.037^{*}$ & $1,625.96 \pm 509.31$ & 0.560 \\
\hline Protein (g/day) & $70.85 \pm 22.10$ & $68.75 \pm 23.29$ & 0.201 & $69.36 \pm 22.77$ & 0.550 \\
\hline Carbohydrate (g/day) & $224.76 \pm 77.79$ & $209.95 \pm 67.82$ & $0.004^{*}$ & $218.05 \pm 81.67$ & 0.447 \\
\hline
\end{tabular}


Table 2 The baseline attributes of the participants with no fall, occasional and recurrent falls (Continued)

\begin{tabular}{|c|c|c|c|c|c|}
\hline Parameters & $\begin{array}{l}\text { Non-fallers } \\
(n=1454)[\text { Prevalence } \\
(\%) \text { or mean } \pm \text { SD] }\end{array}$ & $\begin{array}{l}\text { Occasional fallers } \\
(n=224)[\text { Prevalence } \\
(\%) \text { or mean } \pm \text { SD] }\end{array}$ & $P$-value & $\begin{array}{l}\text { Recurrent fallers } \\
(n=85) \text { [Prevalence } \\
(\%) \text { or mean } \pm \text { SD] }\end{array}$ & $P$-value \\
\hline Sugar (g/day) & $21.51 \pm 15.12$ & $20.42 \pm 14.94$ & 0.331 & $18.51 \pm 12.94$ & 0.077 \\
\hline Fat (g/day) & $52.87 \pm 20.69$ & $51.79 \pm 20.36$ & 0.477 & $53.13 \pm 20.72$ & 0.912 \\
\hline Cholesterol (mg/day) & $158.51 \pm 113.21$ & $162.88 \pm 122.33$ & 0.606 & $172.06 \pm 130.20$ & 0.294 \\
\hline Saturated fat (mg/day) & $8.39 \pm 5.73$ & $8.20 \pm 6.14$ & 0.681 & $9.05 \pm 6.10$ & 0.307 \\
\hline MUFA (g/day) & $8.32 \pm 5.02$ & $8.44 \pm 5.65$ & 0.750 & $8.71 \pm 5.73$ & 0.498 \\
\hline PUFA (g/day) & $5.44 \pm 3.43$ & $5.49 \pm 3.67$ & 0.834 & $5.40 \pm 3.22$ & 0.916 \\
\hline Vitamin D (mg/day) & $0.35 \pm 2.50$ & $0.27 \pm 0.97$ & 0.682 & $0.34 \pm 1.05$ & 0.990 \\
\hline Vitamin E (mg/day) & $12.04 \pm 62.58$ & $14.77 \pm 71.91$ & 0.563 & $4.33 \pm 2.40$ & 0.262 \\
\hline a-tocopherol (mg/day) & $0.45 \pm 1.17$ & $0.51 \pm 1.45$ & 0.506 & $0.54 \pm 1.22$ & 0.495 \\
\hline Sodium (mg/day) & $1,466.23 \pm 979.55$ & $1,401.85 \pm 1068.97$ & 0.545 & $1,466.36 \pm 799.12$ & 0.854 \\
\hline Potassium (mg/day) & $1,510.35 \pm 552.18$ & $1,443.59 \pm 522.91$ & 0.099 & $1,402.60 \pm 534.01$ & 0.083 \\
\hline Calcium (mg/day) & $520.70 \pm 248.06$ & $501.46 \pm 242.21$ & 0.292 & $473.34 \pm 212.43$ & 0.089 \\
\hline Iron (mg/day) & $13.51 \pm 5.41$ & $12.96 \pm 4.84$ & 0.160 & $13.26 \pm 6.10$ & 0.681 \\
\hline Phosphorus (mg/day) & $1,094.54 \pm 418.46$ & $1,081.38 \pm 425.24$ & 0.671 & $1,067.02 \pm 389.09$ & 0.559 \\
\hline Magnesium (mg/day) & $131.86 \pm 64.94$ & $128.51 \pm 68.80$ & 0.489 & $123.00 \pm 63.66$ & 0.227 \\
\hline Zinc (mg/day) & $3.63 \pm 1.99$ & $3.38 \pm 1.60$ & $0.047^{*}$ & $3.40 \pm 1.65$ & 0.310 \\
\hline Selenium ( $\mu \mathrm{g} /$ day) & $23.98 \pm 18.23$ & $22.47 \pm 17.17$ & 0.260 & $23.88 \pm 16.95$ & 0.961 \\
\hline \multicolumn{6}{|l|}{ Biochemical } \\
\hline Hemoglobin (g/L) & $14.20 \pm 2.30$ & $13.64 \pm 1.85$ & $<0.001^{*}$ & $13.42 \pm 2.13$ & $0.008^{*}$ \\
\hline Glucose (mmol/L) & $6.19 \pm 2.28$ & $5.95 \pm 1.69$ & 0.096 & $6.18 \pm 2.00$ & 0.987 \\
\hline Cholesterol (mmol/L) & $5.41 \pm 1.12$ & $5.47 \pm 1.09$ & 0.532 & $5.54 \pm 1.05$ & 0.359 \\
\hline $\mathrm{HDL}(\mathrm{mmol} / \mathrm{L})$ & $1.40 \pm 0.34$ & $1.41 \pm 0.39$ & 0.726 & $1.47 \pm 0.46$ & 0.121 \\
\hline LDL (mmol/L) & $3.34 \pm 1.03$ & $3.36 \pm 0.95$ & 0.806 & $3.42 \pm 0.98$ & 0.523 \\
\hline Triglyceride (mmol/L) & $1.50 \pm 0.76$ & $1.55 \pm 0.76$ & 0.513 & $1.45 \pm 0.63$ & 0.598 \\
\hline Albumin $(\mathrm{g} / \mathrm{L})$ & $42.93 \pm 2.77$ & $42.86 \pm 2.75$ & 0.736 & $42.42 \pm 2.90$ & 0.158 \\
\hline \multicolumn{6}{|l|}{ Cognitive Test } \\
\hline Digit Span & $7.62 \pm 2.42$ & $7.46 \pm 2.37$ & 0.346 & $7.43 \pm 2.40$ & 0.479 \\
\hline MMSE & $23.32 \pm 4.64$ & $22.62 \pm 4.82$ & $0.037^{*}$ & $22.77 \pm 5.16$ & 0.299 \\
\hline MoCA & $19.23 \pm 5.57$ & $18.35 \pm 5.82$ & $0.031^{*}$ & $18.56 \pm 5.88$ & 0.294 \\
\hline Digit Symbol & $5.14 \pm 2.59$ & $4.73 \pm 2.50$ & $0.038^{*}$ & $4.55 \pm 2.29$ & 0.059 \\
\hline \multicolumn{6}{|l|}{ Physical performance } \\
\hline 2-minute step test (number) & $62.44 \pm 25.45$ & $60.70 \pm 24.96$ & 0.350 & $56.31 \pm 32.72$ & 0.104 \\
\hline Chair stand test (number) & $10.05 \pm 2.98$ & $9.60 \pm 3.28$ & $0.039^{*}$ & $8.83 \pm 3.07$ & $<0.001^{*}$ \\
\hline Timed Up and Go test (seconds) & $11.33 \pm 3.56$ & $11.62 \pm 3.49$ & 0.258 & $12.84 \pm 5.28$ & $0.013^{*}$ \\
\hline Dominant handgrip muscle strength test (kg) & $22.59 \pm 7.66$ & $20.82 \pm 7.27$ & $0.002^{*}$ & $19.57 \pm 6.69$ & $0.001^{*}$ \\
\hline
\end{tabular}

Note: BMI Body mass index, HDL High-density lipoprotein, LDL Low-density lipoprotein, MMSE Mini-mental state examination, MoCA Montreal cognitive assessment, MUFA Monounsaturated fatty acids, PUFA Polyunsaturated fatty acids. *Significant differences as compared to non-fallers ( $p<0.05$ )

study was 8.47 and 3.21 per 100 person-years respectively, with no increase with age. Falls prevalence of 15$18 \%[18,19]$ and $27 \%$ over a six-months follow-up period [20] had previously been reported among community-dwelling older Malaysians. The prevalence of recurrent falls is reported as $8.3 \%$ [21].
Despite the apparent association between age and decline in both physical and cognitive functions [5], our results showed that advancing age did not predict the incidence of occasional and recurrent falls. In an agespecific population, the incidence of falls was not agedependent, as opposed to the prevalence of falls [1]. This 
Table 3 Univariate scores for individual predictors of occasional and recurrent falls

\begin{tabular}{|c|c|c|c|c|}
\hline Domains & Variables & $P$-value & $\operatorname{Exp}(B)$ & {$[95 \% \mathrm{Cl}]$} \\
\hline \multicolumn{5}{|l|}{ Occasional falls } \\
\hline \multirow[t]{3}{*}{ Sociodemographic } & Sex (Female) & $0.001^{*}$ & 1.62 & $1.22-2.16$ \\
\hline & Marital status (Single) & $0.036^{*}$ & 2.57 & $1.06-6.23$ \\
\hline & Falls history & $<0.001^{*}$ & 2.42 & $1.76-3.33$ \\
\hline Psychosocial & Depressive symptoms & $0.013^{*}$ & 1.08 & $1.02-1.14$ \\
\hline \multirow[t]{2}{*}{ Body composition } & Fat-free mass & $0.007^{*}$ & 0.97 & $0.96-0.99$ \\
\hline & Skeletal muscle mass & $0.006^{*}$ & 0.96 & $0.93-0.99$ \\
\hline \multirow[t]{3}{*}{ Nutrition } & Energy & $0.037^{*}$ & 1.00 & $1.00-1.00$ \\
\hline & Carbohydrate & $0.009^{*}$ & 1.00 & $1.00-1.00$ \\
\hline & Zinc & $0.089^{*}$ & 0.93 & $0.86-1.01$ \\
\hline Biochemical & Hemoglobin & $0.002^{*}$ & 0.88 & $0.88-0.96$ \\
\hline \multirow[t]{3}{*}{ Cognitive test } & MMSE & $0.037^{*}$ & 0.97 & $0.94-1.00$ \\
\hline & MoCA & $0.031^{*}$ & 0.97 & $0.95-1.00$ \\
\hline & Digit Symbol & $0.039^{*}$ & 0.94 & $0.88-1.00$ \\
\hline \multirow[t]{2}{*}{ Physical performance } & Chair stand test & $0.039^{*}$ & 0.95 & $0.91-1.00$ \\
\hline & Dominant handgrip muscle strength test & $0.002^{*}$ & 0.97 & $0.95-0.99$ \\
\hline \multicolumn{5}{|l|}{ Recurrent falls } \\
\hline \multirow[t]{3}{*}{ Sociodemographic } & Sex (Female) & $0.001^{*}$ & 2.30 & $1.44-3.67$ \\
\hline & Living alone & $0.017^{*}$ & 2.04 & $1.14-3.67$ \\
\hline & Falls history & $<0.001^{*}$ & 4.08 & $2.59-6.42$ \\
\hline \multirow[t]{2}{*}{ Chronic disease } & Stroke & $0.036^{*}$ & 3.21 & $1.08-9.55$ \\
\hline & Joint pain & $0.006^{*}$ & 1.90 & $1.20-3.01$ \\
\hline Psychosocial & Depressive symptoms & $0.001^{*}$ & 1.14 & $1.05-1.24$ \\
\hline \multirow[t]{4}{*}{ Body composition } & Fat mass & $0.039^{*}$ & 1.03 & $1.00-1.05$ \\
\hline & Fat-free mass & $0.025^{*}$ & 0.97 & $0.94-1.00$ \\
\hline & Skeletal muscle mass & $0.020^{*}$ & 0.94 & $0.90-0.99$ \\
\hline & Percentage of body fat & $0.006^{*}$ & 1.03 & $1.01-1.05$ \\
\hline Biochemical & Hemoglobin & $0.008^{*}$ & 0.83 & $0.72-0.95$ \\
\hline \multirow[t]{3}{*}{ Physical performance } & Chair stand test & $<0.001^{*}$ & 0.87 & $0.80-0.94$ \\
\hline & Timed Up and Go test & $0.001^{*}$ & 1.08 & $1.03-1.13$ \\
\hline & Dominant handgrip muscle strength test & $0.001^{*}$ & 0.95 & $0.92-0.98$ \\
\hline
\end{tabular}

Note: MMSE Mini-mental state examination, MoCA Montreal cognitive assessment ${ }^{*} p<0.05$

suggests that the number of falls does not increase with increasing age. It may be possible that cumulatively, the number of falls among older people in any given period of time could be observed due to the consistent addition of new cases. Decline in memory with age might also be another reason that may have affected the ability of older persons to recall falls incidences.

Occasional falls could be accidental and are usually associated with extrinsic factors [22, 23]. In comparison, recurrent falls among older persons are commonly related to multifactorial intrinsic factors suggesting a more complex risk model. Our study findings showed that the identified predictors for both occasional and recurrent falls were different, with the exception of having history of falls and taking longer to complete the chair stand test. Having lower muscle strength and experiencing falls in the past appeared as robust predictors of both occasional and recurrent falls in community-dwelling older persons. Being female, single, having higher depression scale score and lower hemoglobin levels were predictors of occasional falls. While, predictors of recurrent falls included a history of stroke and having higher percentage of body fat.

A history of falls appeared to be the predictor of both occasional and recurrent falls. These findings are consistent with previous reports where having a history of falls was found to be a significant predictor of subsequent falls [24]. Furthermore, our study results showed 
Table 4 Independent predictors for occasional and recurrent falls at 18 months follow-up

\begin{tabular}{|c|c|c|c|c|}
\hline Domains & Variables & $P$-value & $\operatorname{Exp}(B)$ & {$[95 \% \mathrm{Cl}]$} \\
\hline \multicolumn{5}{|l|}{ Occasional falls } \\
\hline \multirow[t]{3}{*}{ Sociodemographic } & Sex (Female) & $0.033^{*}$ & 1.57 & $1.04-2.36$ \\
\hline & Marital status (Single) & $<0.001^{*}$ & 5.31 & $3.36-37.48$ \\
\hline & Falls history & $0.006^{*}$ & 1.86 & $1.19-2.92$ \\
\hline Psychosocial & Depressive symptoms & $0.014^{*}$ & 1.10 & $1.02-1.20$ \\
\hline Biochemical & Hemoglobin & $0.040^{*}$ & 0.90 & $0.81-1.00$ \\
\hline Physical performance & Chair stand test & $0.040^{*}$ & 0.93 & $0.87-1.00$ \\
\hline \multicolumn{5}{|l|}{ Recurrent falls } \\
\hline Sociodemographic & Falls history & $0.002^{*}$ & 2.74 & $1.45-5.19$ \\
\hline Chronic disease & Stroke & $0.003^{*}$ & 8.57 & $2.12-34.65$ \\
\hline Body composition & Percentage of body fat & $0.011^{*}$ & 1.04 & $1.01-1.08$ \\
\hline Physical performance & Chair stand test & $<0.001^{*}$ & 0.87 & $0.77-0.97$ \\
\hline
\end{tabular}

Note: ${ }^{*} p<0.05$

that older women had higher risk of falls as compared to men. Older women having a higher risk of falls has been well established in the literature. Previously, the English Longitudinal Study of Ageing (ELSA) involving 1994 men and 2357 women had reported higher prevalence of falls among women (29.1\%) compared to men (23.5\%) [2]. The number of women admitted to hospital due to falls increased every year from 15,000 in year 2001 to 20, 000 in 2009 [25]. It is also plausible that women have a greater loss in bone mineral density due to menopause and this could be associated with decline in muscle strength and falls.

Depression is associated with occasional falls among community-dwelling older persons as reported previously [26]. Depressive symptoms may affect older persons' mobility and executive function [27]. The causal relationship between depression and falls was not fully explained by adjustment for the medical comorbidities, nutritional, physical and biochemical factors. Use of medications was not adjusted within this study, which may account for the increased risk of falls among individuals with symptoms of depression [28]. Depression in older persons has been attributed to structural brain changes that interfere with cortical-subcortical circuits, basal-ganglia and limbic networks, which in turn affect postural stability leading to the occurrence of falls [29]. Furthermore, antidepressants have been associated with single and recurrent falls $[29,30]$. The proposed mechanisms for antidepressant-related falls include orthostatic hypotension, dizziness, compromised vision and mental confusion [30].

Lower hemoglobin levels increased the risk of occasional falls. Potential mechanisms linking the age-associated decline in hemoglobin and falls include fatigue, reduced muscle strength and muscle quality. The decline in oxygen delivery is attributed to the reduction of hemoglobin levels, whereby hemoglobin functions as an oxygen carrier to skeletal muscles, leading to a reduction in muscle function and declining mobility. This finding was in agreement with the three-year Longitudinal Aging Study Amsterdam demonstrating frequent episodes of falls among older persons with anemia as compared to their non-anemic counterparts [31]. The presence of lower hemoglobin may also reflect underlying nutritional deficiencies or chronic conditions affecting hemoglobin production. Others include undetected causes of hemoglobin loss due to medical conditions such as peptic ulcer disease or malignancy and medications, including ulcer-inducing drugs and those that inhibit marrow function. However, these factors had not been fully accounted for within this study.

The chair stand test, a measure of lower extremity muscle strength, has been demonstrated to be beneficial in determining fall risk [32]. Older persons who had lower chair stand test scores were reported to be associated with a higher risk of fall-related injuries [33]. Moreover, lower extremity weakness was reported to increase the odds of occasional and recurrent falls in older persons [34] since it was associated with abnormal gait, loss of balance, declined mobility, flexibility and functional performance [35]. Besides, strengthening of lower limb muscles has been reported to be effective in preventing falls in older persons [35]. Similarly, we have demonstrated that muscle strength was associated with falls among Malaysian community-dwelling older persons in our earlier pooled data findings [36].

Our study results also showed that the risk of recurrent falls at 18 months was increased among older persons with higher percentage of body fat. One of the probable reasons for this relationship could be due to declined lower extremity muscle strength following excess adiposity, which could affect postural stability and balance [37]. Excess adipose tissue accumulation may also 
lead to dynapenic obesity, a condition linked to a decline in muscle strength, loss of muscle mass and sarcopenic obesity [38, 39]. Recent studies have also linked adiposity with low-grade inflammation, which not only predisposes individuals to osteoarthritis and dementia but also increases the risk of sarcopenia and osteoporosis [40]. As a result, it may lead to impaired mobility and balance and consequently, increase fall risk in older persons. In an observational study involving 164,737 participants between the ages of 19 to 106 years, older persons with obesity had the odd ratio of 1.10 and 1.12 for one fall and two or more episodes of falls respectively [41].

Increased body fat predisposes individuals to underlying medical conditions such as diabetes, hypertension, heart disease, hypercholesterolemia and stroke. Stroke also has been identified as one of the major risk factors of falls and recurrent falls [42]. Stroke survivors tend to develop fear of falls, which is associated with physical and functional decline, decreased quality of life, impaired social interaction, depression and anxiety [43, 44]. Depressive symptoms and loss of dynamic balance have been demonstrated to increase the risk of falls among stroke survivors $[45,46]$.

In our longitudinal study, being single (unmarried) was a risk factor of occasional falls during the 18thmonths follow-up. Single older persons were commonly identified as living alone, experiencing loneliness, depressive symptoms and poor health $[47,48]$. Similarly, these characteristics were associated with increased risk of falls in older persons. Older persons living alone may be having declined physical fitness because of limited participation in physical activity $[49,50]$. Subsequently, having an increased risk of fall-related injuries, mortality and morbidity $[49,50]$.

One of the main limitations of this study is that we obtained retrospective history of falls subjectively. Older persons may have difficulties recalling their fall events in the past 18-months, causing under or over-reporting of the frequency of falls.

\section{Conclusions}

In conclusion, the incidence rates of occasional and recurrent falls in community-dwelling older Malaysian were 8.47 and 3.21 per 100 person-years respectively. Being female, single, having history of falls, higher depression scale, lower hemoglobin levels and lower chair stand test score remained independent predictors of occasional falls. Having history of falls, being a stroke survivor, higher percentage of body fat and lower chair stand test score appeared as recurrent falls predictors in this model. In this study, we identified predictors for prospective falls using a comprehensive, multidomain approach. Further studies are required to determine the value of individualized interventions informed by such an approach to risk factors identification.

\section{Abbreviations}

BMI: Body Mass Index; HDL: High-density lipoprotein; LRGS-TUA: Long-term Research Grant Scheme - Towards Useful Ageing; LDL: Low-density lipoprotein; MMSE: Mini-Mental State Examination; MoCA: Montreal Cognitive Assessment; TUG: Time Up and Go; WAIS: Wechsler Adult Intelligence Scale

\section{Acknowledgements}

We thank all the participants and the research team for their support and assistance rendered in the study. We would like to thank Professor Dr. Michael Fenech, Distinguished Professor, Faculty of Health Sciences, UKM for reviewing and proofreading this manuscript.

\section{Authors' contributions}

DKAS, SS, NFR, RS, DV involved in the conception and study design. TCO and MPT contributed to data analysis and interpretation. TCO drafted the original manuscript. All authors read and approved the final manuscript.

\section{Funding}

This work was supported by the Long-term Research Grant Scheme provided by the Ministry of Education Malaysia (LRGS/BU/2012/UKM-UKM/K/01). The funder had no role in the design of the study; collection, analysis and interpretation of data; and in writing the manuscript.

\section{Availability of data and materials}

The datasets used and/or analyzed during the current study are available from the corresponding author on reasonable request.

\section{Declarations}

Ethics approval and consent to participate

This study is a part of LRGS-TUA longitudinal study, approved by the Medical Research and Ethics Committee of Universiti Kebangsaan Malaysia (UKM 1.5.3.5/244/NN-060-2013). Written informed consent was obtained from study participants. In the case of participants with cognitive decline and not capable of providing ethical consent, informed consent was obtained from their legal guardian, family, or caregiver on their behalf.

\section{Consent for publication}

Not applicable.

\section{Competing interests}

The authors declare that they have no competing interests.

\section{Author details}

${ }^{1}$ Centre for Healthy Ageing and Wellness, Faculty of Health Sciences, Universiti Kebangsaan Malaysia, Jalan Raja Muda Abdul Aziz, 50300 Kuala Lumpur, Malaysia. ${ }^{2}$ Nutrition \& Dietetics Programme, School of Health Sciences, Health Campus, Universiti Sains Malaysia, 16100 Kubang Kerian, Kelantan, Malaysia. ${ }^{3}$ Department of Medicine, Faculty of Medicine, University of Malaya, 50603 Kuala Lumpur, Malaysia. ${ }^{4}$ Ageing and Age-Associated Disorders Research Group, Faculty of Medicine, University of Malaya, 50603 Kuala Lumpur, Malaysia.

Received: 24 September 2020 Accepted: 22 February 2021

Published online: 02 March 2021

\section{References}

1. James SL, Lucchesi LR, Bisignano C, Castle CD, Dingels ZV, Fox JT, et al. The global burden of falls: Global, regional and national estimates of morbidity and mortality from the global burden of disease study 2017. Inj Prev. 2020. https://doi.org/10.1136/injuryprev-2019-043286.

2. Gale CR, Westbury LD, Cooper C, Dennison EM. Risk factors for incident falls in older men and women: the English longitudinal study of ageing. BMC Geriatr. 2018;18(1):117.

3. Florence CS, Bergen G, Atherly A, Burns E, Stevens J, Drake C. Medical costs of fatal and nonfatal falls in older persons. J Am Geriatr Soc. 2018;66(4):693-8.

4. Yeong UY, Tan SY, Yap JF, Choo WY. Prevalence of falls among communitydwelling elderly and its associated factors: A cross-sectional study in Perak, Malaysia. Malays Fam Physician. 2016;11(1):7-14. 
5. Ambrose AF, Paul G, Hausdorff JM. Risk factors for falls among older persons: A review of the literature. Maturitas. 2013;75(1):51-61.

6. Hsu H, Jhan L. Risk factors of falling among the elderly in Taiwan: a longitudinal study. Taiwan Geriatr Gerontol. 2008;3(2):141-54.

7. Paliwal Y, Slattum PW, Ratliff SM. Chronic health conditions as a risk factor for falls among the community-dwelling US older persons: a zero-inflated regression modeling approach. Biomed Res Int. 2017;2017:5146378.

8. Smee DJ, Anson JM, Waddington GS, Berry HL. Association between physical functionality and falls risk in community-living older persons. Curr Gerontol Geriatr Res. 2012;2012:864516.

9. Blackwood J, Martin A. Screening for cognitive impairment as a part of falls risk assessment in physical therapist practice. J Geriatr Phys Ther. 2017;40(4): 197-203.

10. Nash L, Bergin N. Nutritional strategies to reduce falls risk in older people. Nurs Older People. 2018;30(3):20-4.

11. Shahar S, Omar A, Vanoh D, Hamid TA, Mukari SZ, Din NC, et al. Approaches in methodology for population-based longitudinal study on neuroprotective model for healthy longevity (TUA) among Malaysian older persons. Aging Clin Exp Res. 2016;28(6):1089-104.

12. Ibrahim NM, Shohaimi S, Chong HT, Rahman AH, Razali R, Esther E, et al. Validation study of the mini-mental state examination in a Malay-speaking elderly population in Malaysia. Dement Geriatr Cogn Disord. 2009;27(3):247-53.

13. Wechsler $D$. The measurement and appraisal of adult intelligence. 4th ed. Baltimore: Williams \& Wilkins Co; 1958.

14. Razali R, Jean-Li L, Jaffar A, Ahmad M, Shah SA, Ibrahim N, et al. Is the Bahasa Malaysia version of the Montreal Cognitive Assessment (MoCA-BM) a better instrument than the Malay version of the Mini Mental State Examination (M-MMSE) in screening for mild cognitive impairment (MCI) in the elderly? Compr Psychiatry. 2014;55(Suppl. 1):70-5.

15. Strauss E, Sherman EMS, Spreen O. A compendium of neuropsychological tests: administration, norms, and commentary. 3rd ed. New York: Oxford University Press; 2006.

16. Teh EE. Hasanah Che Ismail. Validation of Malay version of geriatric depression scale among elderly inpatients. Universiti Sains Malaysia. 2004. Accessed 20 July 2020.

17. World Health Organization. WHO Global report on falls prevention in older age. Geneva: World Health Organization; 2007.

18. Ibrahim A, Singh DKA, Shahar S. "Timed Up and Go" test: Age, gender and cognitive impairment stratified normative values of older persons. PloS one. 2017:12(10):e0185641.

19. Ibrahim A, Singh DKA, Shahar S, Omar MA. Timed up and go test combined with self-rated multifactorial questionnaire on falls risk and sociodemographic factors predicts falls among community-dwelling older persons better than the timed up and go test on its own. J Multidiscip Healthc. 2017;10:409.

20. Singh DKA, Samah ZA, Shahar S, Omar MA, Shaharudin MI, Chin AV. Sociodemographic characteristics as determinants and physical performance measures as correlates of falls among Malaysian community-dwelling older persons: a prospective study. Top Geriatr Rehabil. 2019:35(2):113-8.

21. Sazlina SG, Chan YM, Hamid TA, Suzana S, Ajit Singh DK. Associations between falls and psychosocial factors, self-rated health, disability and sleep among community dwelling older people in Malaysia. J Clin Gerontol Geriatr. 2018;9(3):85-90.

22. Agudelo-Botero M, Giraldo-Rodríguez L, Murillo-González JC, MinoLeón D, Cruz-Arenas E. Factors associated with occasional and recurrent falls in Mexican community-dwelling older people. PLoS One. 2018;13(2):e0192926.

23. Gassmann KG, Rupprecht R, Freiberger E. Predictors for occasional and recurrent falls in community-dwelling older people. Z Gerontol Geriatr. 2009:42(1):3-10.

24. Tinetti ME, Kumar $C$. The patient who falls: "It's always a trade-off" JAMA. 2010;303(3):258-66.

25. Vitor PR, de Oliveira AC, Kohler R, Winter GR, Rodacki C, Krause MP. Prevalence of falls in elderly women. Acta Ortop Bras. 2015;23(3):158-61.

26. Briggs R, Kennelly SP, Kenny RA. Does baseline depression increase the risk of unexplained and accidental falls in a cohort of community-dwelling older people? Data from The Irish Longitudinal Study on Ageing (TILDA). Int J Geriatr Psychiatry. 2018:33(2):e205-11.

27. Pieruccini-Faria F, Muir-Hunter SW, Montero-Odasso M. Do depressive symptoms affect balance in older persons with mild cognitive impairment? Results from the "gait and brain study. Exp Gerontol. 2018;108:106-11.
28. Takakusaki K. Neurophysiology of gait: from the spinal cord to the frontal lobe. Mov Disord. 2013;28(11):1483-91.

29. Marcum ZA, Perera S, Thorpe JM, Switzer GE, Castle NG, Strotmeyer ES, et al. Antidepressant use and recurrent falls in community-dwelling older persons: Findings from the health $A B C$ study. Ann Pharmacother. 2016;50(7): $525-33$.

30. Quach L, Yang FM, Berry SD, Newton E, Jones RN, Burr JA, et al. Depression, antidepressants, and falls among community-dwelling elderly people: the MOBILIZE Boston study. J Gerontol A Biol Sci Med Sci. 2013;68(12):1575-81.

31. Penninx BW, Pluijm SM, Lips P, Woodman R, Miedema K, Guralnik JM, et al. Late-life anemia is associated with increased risk of recurrent falls. J Am Geriatr Soc. 2005;53(12):2106-11.

32. Applebaum EV, Breton D, Feng ZW, Ta AT, Walsh K, Chassé K, et al. Modified 30-second Sit to Stand test predicts falls in a cohort of institutionalized older veterans. PLoS One. 2017;12(5):e0176946.

33. Shea CA, Ward RE, Welch SA, Kiely DK, Goldstein R, Bean JF. Inability to perform the repeated chair stand task predicts fall-related injury in older primary care patients. Am J Phys Med Rehabil. 2018;97(6):426-32.

34. Moreland JD, Richardson JA, Goldsmith CH, Clase CM. Muscle weakness and falls in older persons: a systematic review and meta-analysis. J Am Geriatr Soc. 2004;52(7):1121-9.

35. Ishigaki EY, Ramos LG, Carvalho ES, Lunardi AC. Effectiveness of muscle strengthening and description of protocols for preventing falls in the elderly: a systematic review. Braz J Phys Ther. 2014;18(2):111-8.

36. Singh DKA, Shahar S, Vanoh D, Kamaruzzaman SB, Tan MP. Diabetes, arthritis, urinary incontinence, poor self-rated health, higher body mass index and lower handgrip strength are associated with falls among community-dwelling middle-aged and older persons: Pooled analyses from two cross-sectional Malaysian datas. Geriatr Gerontol Int. 2019; 19(8):798-803.

37. Neri SGR, Gadelha AB, Correia ALM, Pereira JC, Safons MP, Lima RM. Association between obesity, risk of falls and fear of falling in older women. Rev Bras Cineantropometria Desempenho Hum. 2017;19:450-8.

38. Hita-Contreras F, Martínez-Amat A, Cruz-Díaz D, Pérez-López FR. Osteosarcopenic obesity and fall prevention strategies. Maturitas. 2015;80(2):126-32.

39. Scott D, Sanders KM, Aitken D, Hayes A, Ebeling PR, Jones G. Sarcopenic obesity and dynapenic obesity: 5-year associations with falls risk in middleaged and older persons. Obes (Silver Spring). 2014;22(6):1568-74.

40. Wedell-Neergaard AS, Eriksen L, Grønbæk M, Pedersen BK, KroghMadsen R, Tolstrup J. Low fitness is associated with abdominal adiposity and low-grade inflammation independent of BMI. PLoS One. 2018;13(1):e0190645.

41. Kim SY, Kim MS, Sim S, Park B, Choi HG. Association between obesity and falls among Korean persons: a population-based cross-sectional study. Med. 2016;95(12):e3130.

42. Tan KM, Tan MP. Stroke and falls-clash of the two titans in geriatrics. Geriatr. 2016;1(4):31.

43. Larén A, Odqvist A, Hansson PO, Persson CU. Fear of falling in acute stroke: The fall study of Gothenburg (FallsGOT). Top Stroke Rehabil. 2018;25(4):256-60.

44. Schmid AA, Arnold SE, Jones VA, Ritter MJ, Sapp SA, Van Puymbroeck M. Fear of falling in people with chronic stroke. Am J Occup Ther. 2015;69(3): 6903350020.

45. Cho K, Lee G. Impaired dynamic balance is associated with falling in poststroke patients. Tohoku J Exp Med. 2013;230(4):233-9.

46. Jørgensen L, Engstad T, Jacobsen BK. Higher incidence of falls in long-term stroke survivors than in population controls: Depressive symptoms predict falls after stroke. Stroke. 2002;33(2):542-7.

47. Lim LL, Kua EH. Living alone, loneliness, and psychological well-being of older persons in singapore. Curr Gerontol Geriatr Res. 2011:2011:673181.

48. Robards J, Evandrou M, Falkingham J, Vlachantoni A. Marital status, health and mortality. Maturitas. 2012;73(4):295-9.

49. Langhammer B, Bergland A, Rydwik E. The importance of physical activity exercise among older people. Biomed Res Int. 2018;2018:7856823.

50. Lee W-J, Cheng Y-Y, Liu J-Y, Yang K-C, Jeng S-Y. Living alone as a red flag sign of falls among older people in rural Taiwan. J Clin Gerontol Geriatr. 2011;2(3):76-9.

\section{Publisher's Note}

Springer Nature remains neutral with regard to jurisdictional claims in published maps and institutional affiliations. 